\title{
Efeitos do treinamento físico sobre a pressão arterial, frequência cardíaca e morfologia cardíaca de ratos hiper- tensos
}

\section{Effects of physical training by blood pressure, heart rate and cardiac morphology in hypertensive rats}

Karla F. Goessler ${ }^{1}$, Marli C. Martins-Pinge ${ }^{2}$, Natalia V. da Cunha ${ }^{2}$, Marlusa Karlen-Amarante ${ }^{3}$, Marcos D. Polito ${ }^{1}$

\begin{abstract}
RESUMO
O objetivo desse estudo foi verificar o efeito da natação sobre as alterações morfológicas cardíacas e hemodinâmicas de ratos com hipertensão induzida por L-NAME. Quarenta ratos Wistar foram divididos nos grupos: controle sedentário (CS), controle treinado (CT), sedentário com L-NAME (LS) e treinado com L-NAME (LT). Os animais treinados realizaram natação por até 60 min durante quatro semanas. Os animais dos grupos L-NAME receberam $20 \mathrm{mg} \cdot \mathrm{kg}^{-1}$ também durante quatro semanas. $\mathrm{O}$ grupo LS apresentou maiores valores de PAM $(136,6 \pm 5,1 \mathrm{mmHg})$ comparado ao CS $(107,1 \pm 1,8 \mathrm{mmHg})$. O grupo LT apresentou reduções na PAM comparado ao LS $(121,2 \pm 1,4$ e $136,6 \pm 5,1 \mathrm{mmHg}$, respectivamente). Por outro lado, os LT ainda permaneceram hipertensos comparados ao CT $(121,0 \pm 1,4$ e 107,1 $\pm 1,8 \mathrm{mmHg}$, respectivamente). Em relação à $\mathrm{FC}$, houve bradicardia de repouso para os animais treinados. Os grupos CS e CT não apresentaram alterações no peso relativo e absoluto do coração. Houve aumento do peso absoluto do coração para o grupo LS comparado ao CS e também se observou aumento para o peso relativo e absoluto do coração para o grupo LT comparado ao CT. A análise histológica demonstrou que o treinamento físico pode reduzir a quantidade de lesões provocadas pela administração crônica de $L$ NAME. Conclui-se que a natação foi eficiente em reduzir a PAM de animais hipertensos, mas não reduziu em animais normotensos. Adicionalmente, o treinamento físico não promoveu hipertrofia cardíaca, mas a administração de L-NAME aumentou o peso absoluto e relativo do coração em animais sedentários e treinados.
\end{abstract}

Palavras-Chave: Treinamento/Natação; Óxido Nítrico; Hipertensão; Sistema Cardiovascular.

1. Departamento de Educação Física, Centro de Educação Física e Esportes, Universidade Estadual de Londrina. Programa de Pós-graduação Associado em Educação Física UEM/UEM.

2. Departamento de Ciências Fisiológicas, Centro de Ciências Biológicas, Universidade Estadual de Londrina. Programa de Pós-graduação Multicêntrico em Ciências Fisiológicas - Universidade Estadual de Londrina.

3. Departamento de Fisiologia, Faculdade de Medicina de Ribeirão Preto, Universidade de São Paulo. Programa de Pós-graduação em Fisiologia - Universidade de São Paulo.
Correspondência:

Karla F. Goessler. Universidade Estadual de Londrina, Centro de Educação Física e Esporte. Rodovia Celso Garcia Cid km 380 - Campus Universitário 86051-090 - Londrina, PR - Brasil. Email: karlagoessler@gmail.com Aprovado para publicação em 23/05/2014 


\begin{abstract}
The objective of this study was to investigate the effect of swimming on the cardiac morphological and hemodynamic changes in rats with hypertension induced by L-NAME.. Forty Wistar rats were divided into four groups: sedentary control (SC), trained control (TC), sedentary with L-NAME (LS) and trained with LNAME (LT). The animals in the training groups performed swimming lasting up to 60 min for four weeks. Animals in the L-NAME groups received $20 \mathrm{mg} \cdot \mathrm{kg}^{-1}$ during four weeks. The results showed that animals in the LS group had higher mean arterial pressure $(136.6 \pm 5.1 \mathrm{mmHg})$ compared to CS $(107.1 \pm 1.8 \mathrm{mmHg})$. The LT group showed significant reductions in mean arterial pressure compared to LS (121.2 \pm 1.4 and $136.6 \pm 5.1 \mathrm{mmHg}$, respectively). On the other hand, the LT group animals still remained hypertensive compared to CT group (121.0 \pm 1.4 and $107.1 \pm 1.8 \mathrm{mmHg}$ respectively). In relation to HR, was observed resting bradycardia for the trained animals. The groups CS and CT showed no changes in relative and absolute weight of the heart. An increase in the absolute weight of the heart to the LS group compared to the CS and also observed an increase in the relative and absolute weight for the LT group compared to CT. Histologic analysis showed that exercise training can reduce the amount of damage caused by chronic administration of L-NAME. In conclusion, we observed that mild exercise was effective in reducing mean arterial pressure in hypertensive rats. Additionally, exercise training did not induced cardiac hypertrophy, but the L-NAME increase the absolute and relative weight of the heart in sedentary and trained rats.
\end{abstract}

Keywords: Swimming/Training, Nitric Oxide, Hypertension, Cardiovascular System.

\section{Introdução}

O óxido nítrico (NO) apresenta importante função vasodilatadora, além de promover importantes efeitos vasculares, renais e cardíacos. ${ }^{1,2}$ Desta forma, a inibição da síntese de NO aumenta os valores de repouso de pressão arterial (PA) e frequência cardíaca (FC) ${ }^{3,4}$ Nesse sentido, Ribeiro et al. ${ }^{5}$ foram os primeiros a demonstrar os efeitos hipertensores da inibição do NO pelo L-NAME in vivo. Evidências na literatura demonstram que a diminuição endógena de NO ocorre em pacientes com hipertensão, hipercolesterolemia, diabetes ou arteriosclerose, o que reduz a vasodilatação endotélio-dependente. ${ }^{6}$ Por outro lado, Dos Santos et al. ${ }^{7}$ demonstraram que animais com hipertensão induzida por L-NAME não apresentaram hiperatividade simpática em um período de 2 e 14 dias após o estabelecimento da hipertensão. No entanto, além dos efeitos da inibição de NO sobre os valores de PA e FC, alguns estudos demonstraram que esse tipo de hipertensão, também pode desenvolver alterações morfológicas cardíacas, como áreas de necrose, aumento de áreas de fibrose, apoptose, redução na angiogênese cardíaca e como consequência a hipertrofia patológica. ${ }^{8}$ Além disso, nesse modelo de hipertensão, além da diminuição da atividade da NO sintase, um aumento da carga oxidativa também pode contribuir para a redução da biodisponibilidade de NO e, consequentemente, promover a hipertensão. ${ }^{9}$ Por outro lado, as alterações morfológicas e da PA e FC proporcionadas pelo bloqueio do NO podem ser re- vertidas com o exercício físico. Estudos que relacionaram o bloqueio de NO com o exercício físico aeróbio em ratos demonstraram que os animais que tiveram o bloqueio da síntese de $\mathrm{NO}$ e realizaram exercício apresentaram redução da $\mathrm{PA}^{4,10}$ e bradicardia $^{3,4,10}$ em relação aos animais sedentários. Adicionalmente, foi demonstrado que o treinamento físico pode apresentar um efeito protetor contra os danos oxidativos e a lesão renal causados pela hipertensão. ${ }^{11}$

Assim, os objetivos desse estudo foram verificar o efeito da hipertensão induzida por L-NAME nas alterações morfológicas cardíacas e o papel do exercício físico na evolução dessas alterações. Além disso, avaliar as respostas hemodinâmicas em resposta a indução da hipertensão pelo L-NAME e ao exercício físico. O protocolo de treinamento físico utilizado no presente estudo (natação), foi testado em estudos anteriores e demonstrou ser eficiente em promover bradicardia de repouso e atenuar a atividade nervosa simpática em animais normotensos. ${ }^{12}$ Nesse contexto, também se caracterizou como um dos objetivos do presente estudo verificar se esse protocolo de treinamento também é eficiente em promover adaptações morfológicas e hemodinâmicas em animais com hipertensão induzida por L-NAME.

\section{Métodos}

\section{Animais}

Foram obtidos 40 ratos Wistar, com peso aproximado de 200-250 g provenientes do Biotério Cen- 
tral do Centro de Ciências Biológicas da Universidade Estadual de Londrina. O projeto foi aprovado pelo Comitê de Ética e Pesquisa em Experimentação Animal (n¹0/11) da mesma instituição.

Os animais foram divididos em quatro grupos: controle sedentários (CS), controle treinados (CT), tratados com L-NAME sedentários (LS) e tratados com L-NAME treinados (LT). Os animais foram mantidos em gaiolas coletivas (cinco animais por gaiola) em temperatura constante de $21 \pm 2^{\circ} \mathrm{C}$, com ciclo claro/escuro de $12 \mathrm{~h}$ e alocados no biotério do Departamento de Ciências Fisiológicas por um período de adaptação por uma semana antes de iniciar os experimentos. Os animais tiveram livre acesso a água potável e ração normocalórica $\left(\mathrm{Nuvilab}^{\circledR}\right)$ e foram pesados diariamente em balança analógica $\left(\right.$ Micronal $^{\circledR}$ ) antes de iniciar o treinamento.

\section{Protocolo de Treinamento}

Os animais realizaram o treinamento físico por natação coletiva em um tanque de vidro $(100 \times 60$ x $50 \mathrm{~cm}$ ) com água a $31 \pm 1^{\circ} \mathrm{C}$ e profundidade de $40 \mathrm{~cm}$ de água. O treinamento ocorreu sempre no período da manhã (09:00h - 13:00h). Após cada sessão de natação, os animais foram colocados em caixas para secagem e aquecimento. Depois de secos, retornavam ao biotério em suas caixas moradia.

Foi utilizado o protocolo previamente utilizado por Martins-Pinge et al. ${ }^{12}$ O treinamento teve duração de quatro semanas, totalizando 20 sessões. A primeira semana correspondeu ao período de adaptação ao treinamento, com aumento gradual do período de natação iniciando com 15 min no primeiro dia, seguido de 30 min no segundo dia, 45 min no terceiro dia e 60 min a partir do quarto dia. A partir do quarto dia, todas as próximas sessões tiveram duração de 60 min. $\mathrm{O}$ volume de treino teve aumento gradual a partir do tempo, não sendo utilizada nenhuma sobrecarga adicional além do próprio peso do animal para aumento de intensidade. Durante o período de treinamento, o grupo sedentário teve seu peso avaliado paralelamente aos animais submetidos ao treinamento físico.

\section{Administração de L-NAME ou Solução Salina}

Para os grupos tratados com L-NAME, foram administrados $20 \mathrm{mg} \cdot \mathrm{kg}^{-1}$ da droga, uma vez por dia, sete dias por semana, durante quatro semanas, por via intraperitoneal. Para os grupos controle, foi administrado o mesmo volume de solução salina estéril. O período de quatro semanas de administração de LNAME ou salina ocorreu paralelamente ao treinamento. A aplicação da droga ou salina ocorreu todos os dias aproximadamente no mesmo horário (16:00 h) para todos os grupos.

\section{Registro da Pressão Arterial e Frequência Cardíaca}

Após o último dia de treinamento, os animais foram submetidos à cirurgia sob anestesia de tribromoetanol $(2,5 \%)$ por via intraperitoneal para implantação de cânulas na artéria femoral com o objetivo de monitorização direta da PA.

As cânulas foram constituídas por segmento de polietileno PE-10 $(4 \mathrm{~cm})$ soldado a um segmento de polietileno PE-50 $(15 \mathrm{~cm})$, previamente preenchidas com solução salina $0,9 \%$ e anticoagulante $(15 \mathrm{U} / \mathrm{mL}$ de heparina em solução salina) e ocluídos.

Uma incisão de $1,0 \mathrm{~cm}$ foi realizada na fossa ilíaca direita do animal e a artéria femoral foi dissecada. Uma linha de costura foi utilizada para isolar a região distal da artéria femoral e uma pinça hemostática foi nela colocada, obstruindo temporariamente o fluxo arterial. Em seguida, um pequeno corte foi feito na artéria femoral e a cânula introduzida e cuidadosamente fixada na artéria. Após esse procedimento, as cânulas foram exteriorizadas na região dorsal do animal através de um trocáter por via subcutânea e fixadas à pele por sutura cirúrgica. Os animais foram mantidos em caixas individuais durante todo o período pós-operatório, permitindo a recuperação dos efeitos anestésicos por um período de 24 horas.

Após o período pós-operatório, a cânula arterial do animal foi acoplada a um transdutor de pressão (Powerlab MLT0380) e conectado a um sistema de registro computadorizado (Powerlab/ADInstruments). Foi realizado o registro com os animais acordados e com livre movimentação por um período de 30 min. Durante o período de registro, os animais foram mantidos em caixas individuais em ambiente silencioso. Para obtenção dos valores de PAM e FC, foi selecionada a média dos $30 \mathrm{~min}$ de registro de cada animal.

\section{Morfologia Cardíaca}

Após o registro, os animais foram sacrificados por inalação de éter dietílico e o coração foi imediatamente retirado e pesado para obtenção do peso absoluto e relativo. O peso relativo do coração (peso do coração x $100 \div$ peso do animal) foi referido como indicativo de hipertrofia cardíaca. ${ }^{13}$ 
Para a análise histológica, foram realizados procedimentos de rotina para coloração em hematoxilina e eosina para identificação de áreas de edema, necrose, infiltrado inflamatório e diâmetro dos cardiomiócitos e em picrossirus red, para a determinação das áreas ocupadas por colágeno no miocárdio, sendo as lâminas analisadas em microscópio óptico. Foram fotografados 10 campos por lâmina de cada animal, sendo os campos determinados de forma aleatória. As imagens foram capturadas por um sistema de captura de imagens digital Moticam $^{\circledR}$ (Motic Company, Xiamen, China) e analisadas em Software Motic Plus 2.0 ${ }^{\circledR}$ (Motic Company, Xiamen, China). Para a determinação do diâmetro dos cardiomiócitos, foi selecionada uma lâmina por animal e traçada uma reta perpendicular ao eixo das células e avaliado o diâmetro das fibras que cortavam a reta. Como critério, foi estabelecido o mínimo de 10 e o máximo de 20 células para essa análise. Os critérios adotados para a análise do grau de lesão, assim como as variáveis analisadas, estão descritos na Tabela 1 .

\section{Análise Estatística}

Os resultados de PAM, FC, peso absoluto e relativo do coração estão expressos em média e erro padrão da média. Para verificar a homogeneidade e normalidade dos dados, foi feito o teste de Levene e Shapiro-Wilk, respectivamente. Foi utilizado o teste ANOVA de duas entradas para a comparação da PAS, PAD, PAM, FC, peso corporal, peso absoluto e relativo do coração e diâmetro dos cardiomiócitos, seguido do teste post-hoc LSD. Na análise de células inflamatórias, os dados estão apresentados em quantidades de campos com lesão, e o teste do Qui-Quadrado foi utilizado para comparar as frequências de lesão entre os grupos. A severidade da lesão foi descrita de acordo com critérios descritos na Tabela 1. Foi adotado o nível de significância de $P<0,05$ e os resultados foram processados no programa estatístico SPSS 17.0.

\section{Resultados}

\section{Variáveis Cardiovasculares: PAS, PAD, PAM e FC}

A Figura 1 apresenta os valores de PAS (A), PAD (B), PAM (C) e FC (D) para todos os grupos. A administração crônica de L-NAME, durante quatro semanas, foi eficiente em induzir a hipertensão, pois o grupo LS apresentou maiores valores de PAS, PAD e PAM comparados aos animais CS $(P<0,001)$. Além disso, o protocolo de natação por quatro semanas reduziu a PAS $(\mathrm{P}=0,04)$, a PAD $(\mathrm{P}=0,02)$ e a PAM dos animais hipertensos (LS vs. LT; $P=0,02$ ). No entanto, os animais LT, mesmo apresentando menores valores de PAS, PAD e PAM quando comparados aos sedentários, ainda permaneceram com valores elevados de PAS $(\mathrm{P}<0,01)$ e PAM $(\mathrm{P}=0,01)$ em relação ao grupo $\mathrm{CT}$ e ao grupo CS PAS $(\mathrm{P}<0,01), \mathrm{PAD}(\mathrm{P}=0,03)$ e PAM $(\mathrm{P}<0,01)$, porém na comparação entre $\mathrm{CT}$ e LT não houve diferença significativa para a PAD $(\mathrm{P}=0,10)$. Para a FC, o grupo CT apresentou bradicardia quando comparado ao CS $(\mathrm{P}=0,003)$, sendo esse mesmo comportamento observado para o grupo LT quando comparado ao LS $(\mathrm{P}=0,02)$.

\section{Morfologia Cardíaca: Peso corporal final, peso absoluto e relativo do coração e diâ- metro dos cardiomiócitos}

A Tabela 2 apresenta os valores correspondentes ao peso corporal final, aos pesos absoluto e relativo do coração e ao diâmetro dos cardiomiócitos e a Figura 2 demonstra como o diâmetro dos cardiomiócitos foi avaliado. Entre os grupos tratados com solução salina, não houve diferença no peso corporal final CS e CT $(\mathrm{P}=0,33)$. No entanto, nos grupos tratados com L-NAME, o grupo LT apresentou menor peso corporal comparado ao grupo LS $(\mathrm{P}<0,01)$. Ainda em

Tabela 1. Critérios de classificação do grau de lesão (edema, neutrófilos e necrose) do músculo cardíaco.

\begin{tabular}{lllll}
\hline & \multicolumn{1}{c}{ Ausência de lesão } & \multicolumn{1}{c}{ Lesão leve } & \multicolumn{1}{c}{ Lesão Moderada } & Lesão acentuada \\
\hline $\begin{array}{l}\text { Dissociação de } \\
\text { fibras musculares } \\
\text { (edema) }\end{array}$ & $\begin{array}{l}\text { Nenhum espaço com } \\
\text { edema por campo }\end{array}$ & $\begin{array}{l}\text { Um espaço com edema } \\
\text { por campo }\end{array}$ & $\begin{array}{l}\text { Dois-quatro espaços } \\
\text { com edema por campo }\end{array}$ & $\begin{array}{l}>\text { cinco espaços com } \\
\text { edema por campo }\end{array}$ \\
$\begin{array}{l}\text { Infiltrado } \\
\text { Neutrofílico }\end{array}$ & $\begin{array}{l}\text { Nenhum neutrófilo por } \\
\text { campo }\end{array}$ & $\begin{array}{l}\text { Um-dez neutrófilos } \\
\text { por campo }\end{array}$ & Onze-vinte neutrófilos & $>\begin{array}{l}>\text { vinte neutrófilos } \\
\text { por campo }\end{array}$ \\
$\begin{array}{l}\text { Necrose de fibras } \\
\text { musculares }\end{array}$ & $\begin{array}{l}\text { Nenhuma fibra com } \\
\text { necrose por campo }\end{array}$ & $\begin{array}{l}\text { Uma fibra com necrose } \\
\text { por campo }\end{array}$ & $\begin{array}{l}\text { Duas-três fibras com } \\
\text { necrose por campo }\end{array}$ & $\begin{array}{l}>\text { quatro fibras com } \\
\text { necrose por campo }\end{array}$ \\
\hline
\end{tabular}



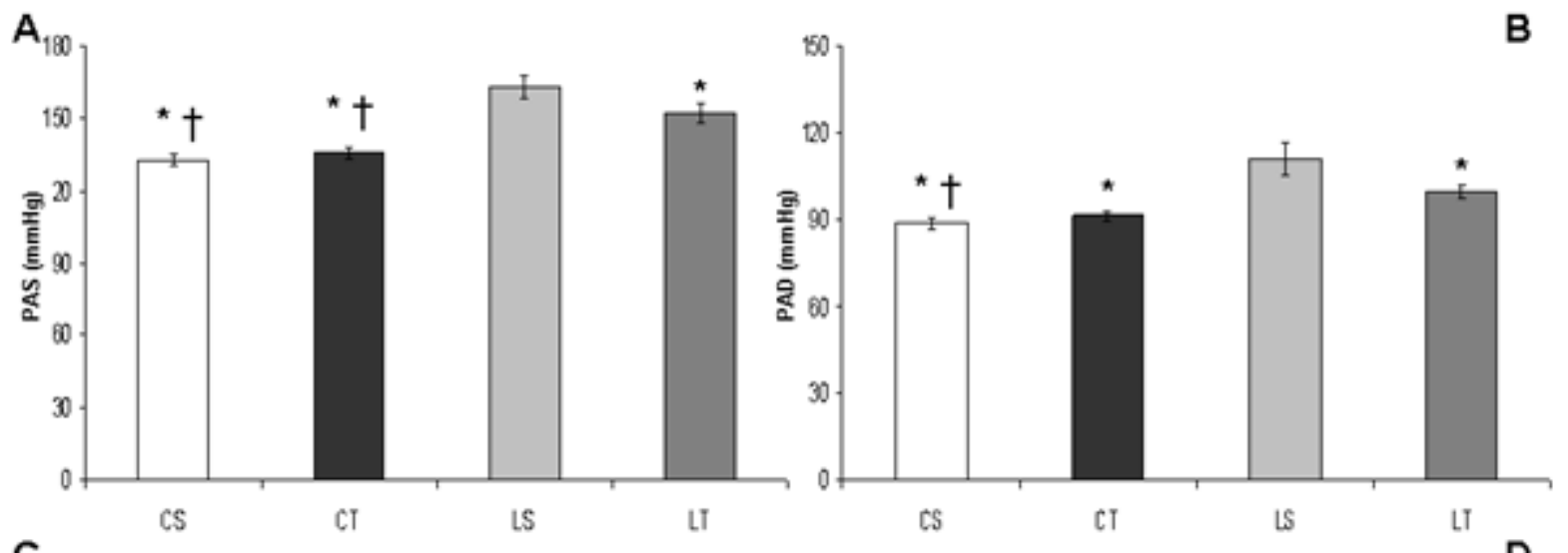

C
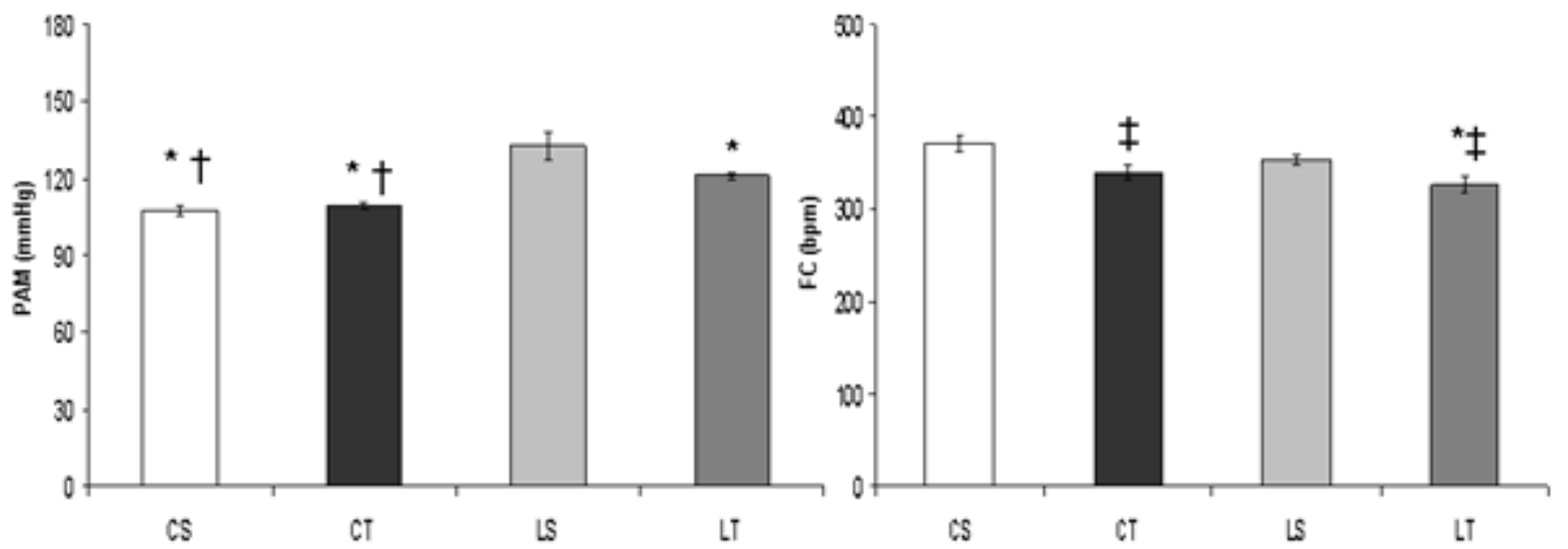

Figura 1. Valores apresentados em média e erro padrão da média para pressão arterial sistólica (A), pressão arterial diastólica (B), pressão arterial média $(C)$ e frequência cardíaca $(D)$ para os grupos sedentários e treinados, tratados com L-NAME ou solução salina. CS: controle sedentário, CT: controle treinado, LS: L-NAME sedentário. *Diferenças em relação ao grupo LS; † Diferenças em relação ao grupo LT; ‡Diferenças em relação ao grupo CS.

relação ao peso corporal, na comparação entre normotensos e hipertensos os grupos CS e CT apresentaram maior peso corporal em relação ao LT $(\mathrm{P}<0,01)$, mas não apresentaram diferenças significativas quando comparados ao LS. Não houve diferença para o peso absoluto e relativo do coração entre os grupos CS e CT ( $\mathrm{P}=0,95$ e $\mathrm{P}=0,55$, respectivamente). No entanto, o peso absoluto do coração foi maior para o grupo LS em relação ao CS $(\mathrm{P}=0,03)$, porém não foi encontrada diferença para o peso relativo entre esses grupos $(\mathrm{P}=0,18)$. No grupo LT foram encontrados maiores valores para o peso absoluto e relativo do coração comparado CT $(\mathrm{P}<0,01)$, demonstrando um possível efeito de hipertrofia cardíaca devido ao LNAME. Ainda na comparação entre normotensos e hipertensos, como esperado, o grupo CT apresentou menor peso absoluto comparado ao LS $(\mathrm{P}=0,04)$, sem apresentar diferença significativa para o peso relati- vo $(\mathrm{P}=0,45)$. Entre os grupos tratados com L-NAME, houve diferença apenas para o peso relativo $(\mathrm{P}=0,01)$, sendo maior para o grupo LT comparado ao LS.

Considerando que houve diferença significativa entre os grupos para o peso corporal, foi realizada a análise do diâmetro dos cardiomiócitos, que confirmou a hipertrofia cardíaca para o grupo LS comparado ao CS $(\mathrm{P}=0,02)$ e ao $\mathrm{CT}(\mathrm{P}=0,01)$, porém sem diferença significativa comparado ao LT $(\mathrm{P}=0,40)$. Esses resultados sugerem que a hipertensão induzida por L-NAME induziu a hipertrofia cardíaca, e que o treinamento físico não foi eficiente em atenuá-la.

\section{Morfologia Cardíaca: Colágeno Cardíaco}

A figura 3 demonstra a comparação da porcentagem de colágeno cardíaco entre os grupos. Não houve diferença entre os grupos CS e CT $(\mathrm{P}=0,82)$ e entre os grupos LS e LT $(\mathrm{P}=0,12)$. No entanto, o grupo 
Tabela 2. Média e erro padrão da média do peso corporal final, peso absoluto e peso relativo do coração dos grupos CS, CT, LS e LT.

\begin{tabular}{lcccc}
\hline Grupos & $\begin{array}{c}\text { Peso Corporal } \\
\text { Final } \\
(\mathbf{g})\end{array}$ & $\begin{array}{c}\text { Peso } \\
\text { absoluto } \\
(\mathbf{m g})\end{array}$ & $\begin{array}{c}\text { Peso relativo } \\
(\mathbf{m g} / \mathbf{g})\end{array}$ & $\begin{array}{c}\text { Diâmetro dos } \\
\text { cardiomiócitos } \\
(\boldsymbol{\mu m})\end{array}$ \\
\hline CS & $347 \pm 4 \dagger$ & $0,99 \pm 0,01^{* \dagger}$ & $0,28 \pm 0,005 \dagger$ & $8,1 \pm 0,9^{*}$ \\
CT & $337 \pm 7 \dagger$ & $0,99 \pm 0,02^{* \dagger}$ & $0,29 \pm 0,005 \dagger$ & $7,8 \pm 0,4^{*}$ \\
LS & $354 \pm 9 \dagger$ & $1,08 \pm 0,04$ & $0,30 \pm 0,01 \dagger$ & $10,3 \pm 0,7$ \\
LT & $311 \pm 8$ & $1,16 \pm 0,02$ & $0,37 \pm 0,01$ & $9,6 \pm 0,3$ \\
\hline
\end{tabular}

${ }^{*} \mathrm{P}<0,05$ vs. LS; $\uparrow \mathrm{P}<0,05$ vs. LT.

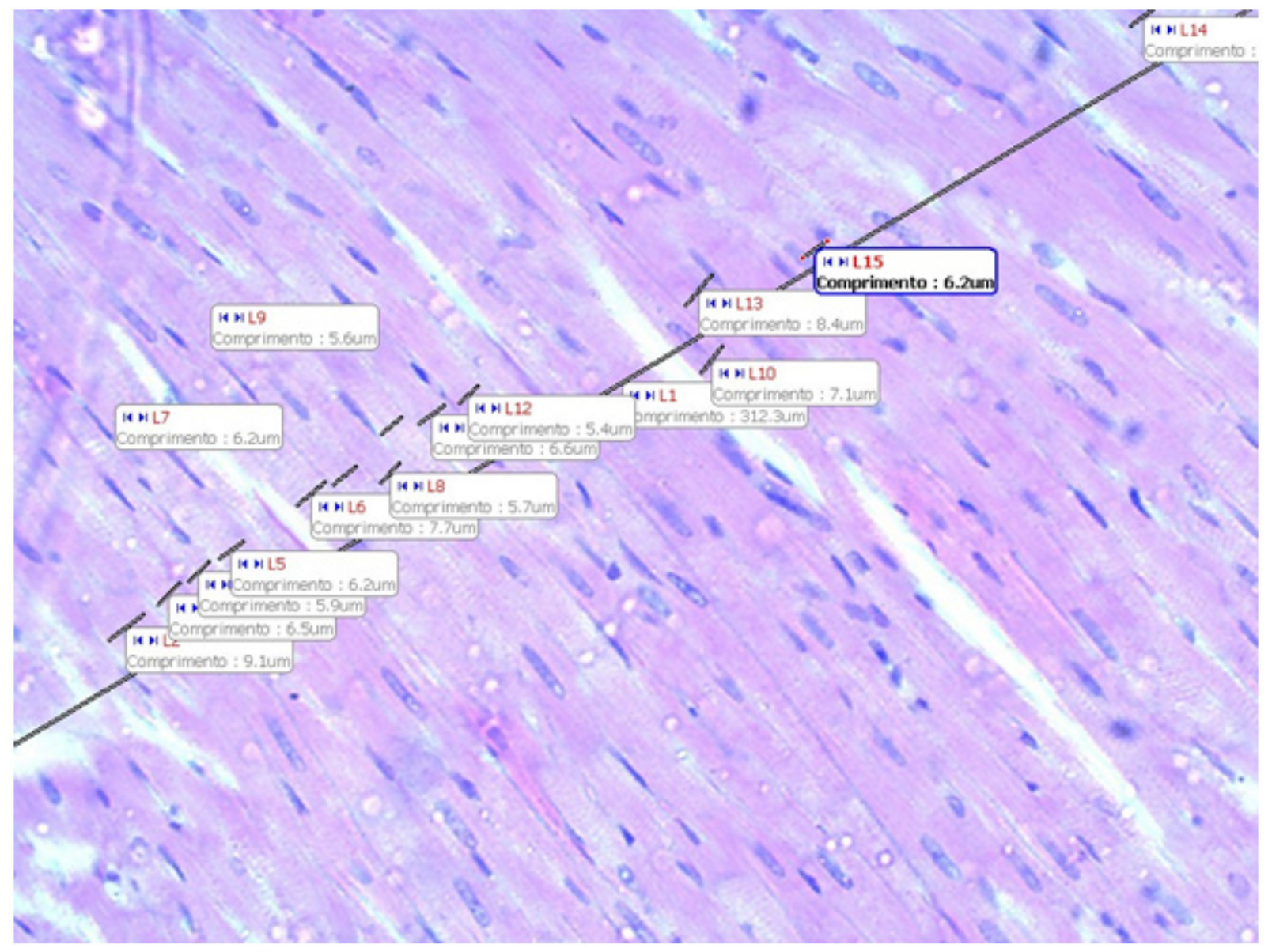

Figura 2. Análise do diâmetro dos cardiomiócitos para avaliação de hipertrofia cardíaca.

LS apresentou maior porcentagem de colágeno comparado ao $\mathrm{CS}(\mathrm{P}<0,01)$ e ao $\mathrm{CT}(\mathrm{P}<0,01)$. Esses achados confirmam a hipótese de que a hipertrofia cardíaca promovida pelo L-NAME é patológica.

\section{Morfologia Cardíaca: Análise Histológica}

A Tabela 3 demonstra a análise de campos com lesão (edema, necrose, neutrófilos e macrófagos) no músculo cardíaco entre os grupos e a Figura 4 ilustra os campos de lesão. Não foram encontradas áreas de necrose em nenhum dos grupos experimentais. O grupo LS, apresentou uma maior quantidade de campos com edema e neutrófilos comparado aos demais grupos (Pd"0,01). O grupo LS, apresentou também mais áreas contendo macrófagos comparado ao grupo CS $(\mathrm{P}<0,01)$, e da mesma forma, o grupo LT apresentou mais áreas com macrófagos comparado ao CT $(\mathrm{P}<0,01)$. 


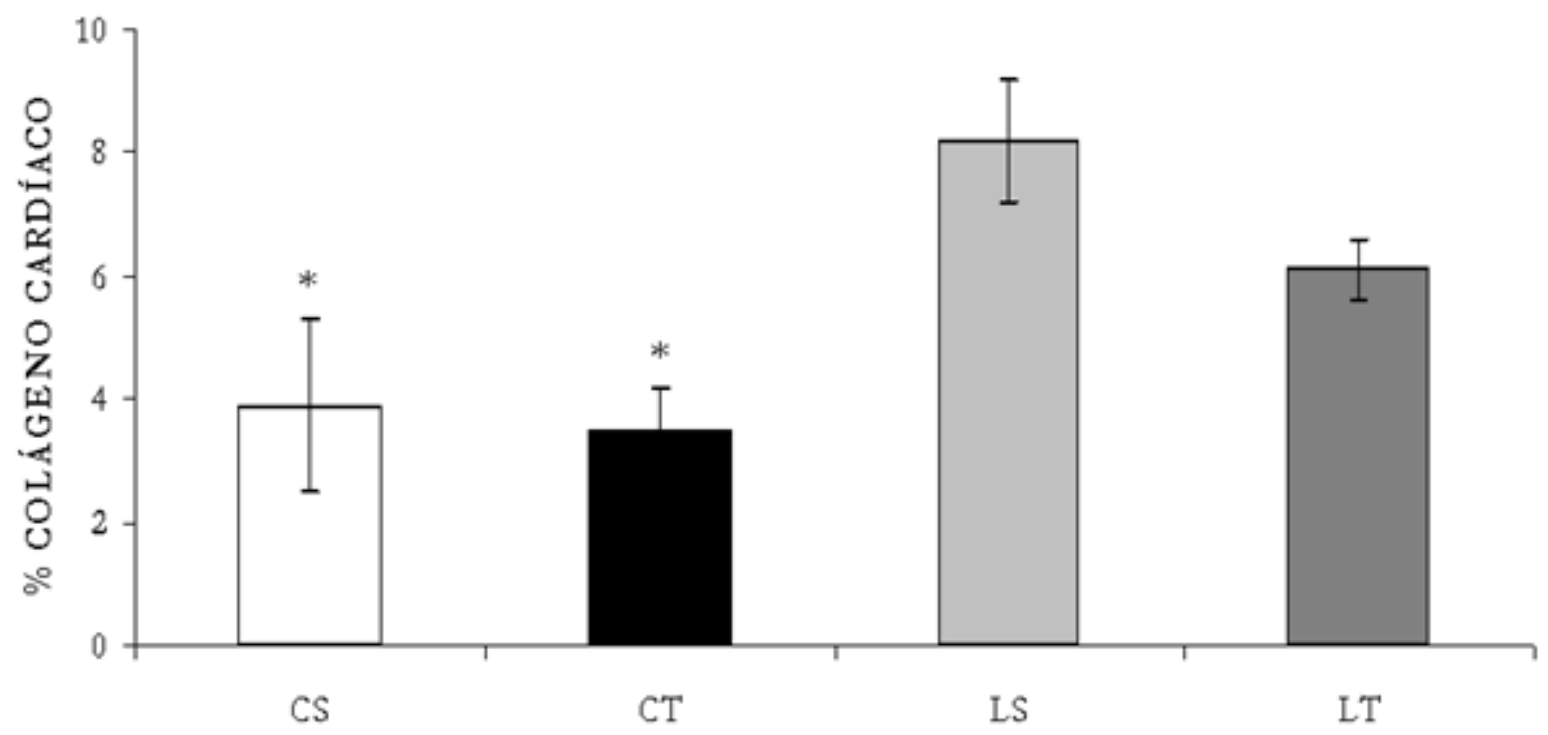

Figura 3. Comparação da porcentagem de colágeno cardíaco entre os grupos sedentários e treinados, tratados com L-NAME ou solução salina. CS: controle sedentário, CT: controle treinado, LS: L-NAME sedentário. *Diferenças em relação ao grupo LS.

Em relação a severidade dessas lesões, as quais foram classificadas de acordo com a Tabela 1, foi possível observar que o grau de edema encontrado no grupo LS foi leve. Além disso, os campos encontrados com a presença de neutrófilos, também foram classificados como leve para todos os grupos, sendo o mesmo encontrado para as áreas com macrófagos, exceto para o grupo CT, o qual apresentou ausência de macrófagos.

\section{Discussão}

A administração de L-NAME (20 mg.kg ${ }^{-1}$ i.p) durante quatro semanas promoveu aumento da PAM de ratos sedentários e treinados comparados aos animais que receberam salina. Além disso, o treinamento físico atenuou a hipertensão induzida por L-NAME, mas esses animais continuaram hipertensos em relação aos controles. Os animais que receberam solução salina não apresentaram redução na PAM com o treinamento físico. Esse resultado está de acordo com estudos anteriores em seres humanos ${ }^{14,15}$, que demonstram que a magnitude da redução da PA promovida pelo exercício físico depende do grau da hipertensão, ou seja, quanto mais hipertenso, maior será o efeito hipotensor do treinamento físico.

Os estudos presentes na literatura são divergentes em relação ao tempo de administração e quantidade de L-NAME necessária para promover hipertensão. ${ }^{3,4,9,16-20} \mathrm{Em}$ alguns estudos ${ }^{3,4}$ foi demonstrado que a administração de L-NAME por uma semana (70 mg. $\mathrm{kg}^{-1}$ - diluída em água) promoveu hipertensão comparado aos animais do grupo controle. No entanto, outro estudo demonstrou que dois e sete dias

Tabela 3. Análise morfológica em relação aos campos com lesão (edema, necrose, neutrófilos e macrófagos) de um total de 50 campos analisados para cada grupo.

\begin{tabular}{lcccc}
\hline Grupos & Edema & Necrose & Neutrófilos & Macrófagos \\
\hline CS & $0^{*}$ & 0 & $3^{*}$ & $3^{*}$ \\
CT & $0^{*}$ & 0 & $3^{*}$ & $0 * \dagger+$ \\
LS & 8 & 0 & 18 & 17 \\
LT & $0^{*}$ & 0 & $7^{*}$ & 9 \\
\hline
\end{tabular}

${ }^{*} \mathrm{P}<0,05$ vs. LS; $† P<0,05$ vs. LT; $\ddagger$ vs. CS. 


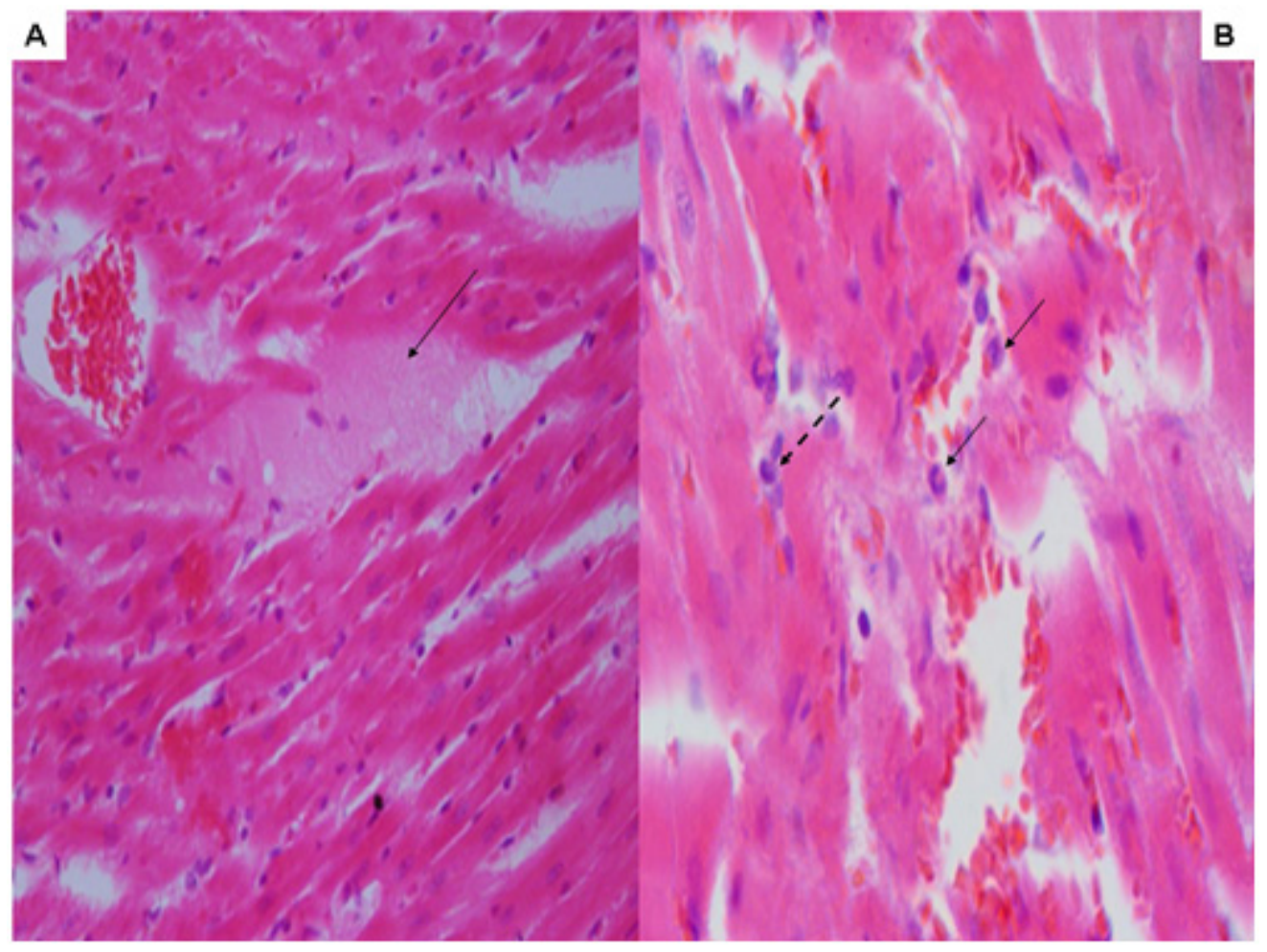

Figura 4. Demonstração de áreas de edema (A) e infiltrado inflamatório (B).

de administração de L-NAME $(20 \mathrm{mg} / \mathrm{kg} / \mathrm{dia}$ gavagem) são suficientes para aumentar a PAM de ratos anestesiados e não anestesiados. ${ }^{21}$ Outros estudos envolvendo maiores concentrações de L-NAME $15 \mathrm{mg} \cdot \mathrm{kg}^{-1} \cdot \mathrm{dia}^{-1(16)}, 40 \mathrm{mg} \cdot \mathrm{kg}^{-1} \cdot \mathrm{dia}^{-1}(9,18,19), 60 \mathrm{mg} \cdot \mathrm{kg}$ ${ }^{1}$ dia $^{-1}{ }^{(20)}$ e com maior tempo de administração (de três a sete semanas) também encontraram aumento na PA. Além disso, nesses estudos citados, observouse que o aumento da PA é progressivo. Na maioria dos estudos, a administração do L-NAME foi por diluição em água e disponibilizada á vontade $3,4,9,16,18$ ${ }^{20} \mathrm{e}$ um estudo administrou a droga por gavagem. ${ }^{17}$ Uma limitação da administração de drogas dissolvidas em água é a falta de controle da ingestão. Por isso, a via de administração adotada em nosso estudo foi injeção intraperitoneal, reduzindo possíveis diferenças nas concentrações ingeridas por cada animal.

Além das diferenças no protocolo de indução de hipertensão por L-NAME, também existem diferenças no protocolo de treinamento entre os estudos. Entre essas diferenças está o tipo de exercício realizado - esteira ${ }^{3,21-23}$ ou natação ${ }^{4,10}$ - e tempo de treinamento - quatro semanas ${ }^{23}$, seis semanas ${ }^{24}$, oito semanas $^{4,21,22}$ e 10 semanas. $^{3}$ No presente estudo, foi utilizado o protocolo de natação durante quatro se- manas, por ter sido utilizado previamente em nosso laboratório ${ }^{12}$ e ter promovido condicionamento cardiovascular em repouso.

Considerando as diferenças quanto ao proto-

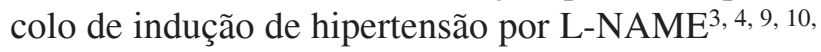
${ }^{16-20}$ e do treinamento físico ${ }^{4,21-23,25}$ o objetivo inicial de estudo foi testar se um protocolo de indução de hipertensão e de natação por quatro semanas, conduzidos simultaneamente seriam eficientes em promover a hipertensão (L-NAME) e/ou promover benefícios em animais possivelmente hipertensos e normotensos (treinamento de natação). Nesse sentido, o estudo de Souza et al. ${ }^{10}$ também analisou variáveis semelhantes as analisadas em nosso estudo, porém com o objetivo de verificar se um melhor condicionamento físico protegeria o sistema cardiovascular contra os danos promovidos pela hipertensão, sendo que esta foi induzida apenas na última semana de treinamento. Em uma perspectiva diferente, nosso estudo testou os dois protocolos (hipertensão e treinamento) partindo de uma situação onde os animais de todos os grupos apresentavam características semelhantes.

Diferente de outros estudos ${ }^{3,4}$, os quais verificaram o efeito do treinamento prévio em atenuar a hipertensão, nosso objetivo foi verificar o efeito do 
treinamento físico de curta duração e sem sobrecarga em uma situação concomitante a indução da hipertensão. Dessa forma, o protocolo de treinamento utilizado foi eficiente em promover a bradicardia de repouso em animais treinados normotensos, como demonstrado anteriormente ${ }^{12} \mathrm{e}$, além disso, o grupo LT também apresentou redução da FC comparado ao LS. Uma das explicações para esse comportamento da FC pode ser melhora no controle autonômico. Nesse sentido, semelhante aos nossos resultados, Souza et al. ${ }^{4}$ encontraram que o bloqueio da síntese de NO provocou alterações no sistema nervoso autonômico em animais sedentários e que animais treinados apresentaram bradicardia basal como melhor controle autonômico. Comparado com outros estudos ${ }^{3,4,10}$, o período de treinamento (4 semanas) é relativamente pequeno, o que pode justificar a ausência de hipertrofia cardíaca nos animais treinados. No entanto, além da hipertrofia cardíaca, outros marcadores de treinamento físico aeróbio, como a concentração de lactato, atividade da enzima citrato sintase, entre outros, devem ser investigados em estudos futuros, a fim de caracterizar este protocolo de treinamento.

Ainda em relação à hipertrofia cardíaca, apesar observarmos maior peso absoluto e relativo do coração para os animais do grupo LT comparado ao CT, o que indicaria uma possível hipertrofia promovida pela hipertensão e não pelo treinamento físico, tendo em vista que não foram observadas diferenças nessas variáveis entre os grupos CS e CT, temos que considerar as diferenças no peso corporal entre os grupos. Considerando a fragilidade desta análise devido à essas diferenças, foi verificado o diâmetro dos cardiomiócitos, para identificação de possível hipertrofia promovida pelo treinamento físico ou pela hipertensão. Com isso, observamos que o grupo LS apresentou maior diâmetro de cardiomiócitos comparado aos grupos CS e CT, sendo essas as únicas diferenças encontradas. Esses resultados nos indicam que mesmo o grupo LT apresentando maior peso absoluto e relativo do coração, não houveram diferenças significativas em relação ao diâmetro dos cardiomiócitos comparado aos demais grupos, o que sugere a ausência de hipertrofia para esse grupo. Por outro lado, o grupo LS apesar de não apresentar diferenças quanto ao peso relativo do coração, demonstrou hipertrófico quando comparado com os grupos CS e CT. Com esses resultados, sugerimos que o protocolo de hipertensão induzida por L-NAME utilizado neste estudo promoveu hipertrofia cardíaca e que o treina- mento físico não foi capaz de reverter essa hipertrofia, tendo em vista que não houve diferença para o diâmetro dos cardiomiócitos entre LS e LT $(\mathrm{P}=0,40)$.

Além dos cardiomiócitos, os fibroblastos também compõem o volume celular do miocárdio, sendo que a complacência da parede ventricular é dependente da quantidade, distribuição e composição das fibras de colágeno presentes no miocárdio. Dessa forma, em determinados tipos de hipertrofia, como as patológicas, pode ser observado um aumento na quantidade de fibras colágenas de maior rigidez, o que pode promover alterações no processo de relaxamento do coração. Por outro lado, na hipertrofia fisiológica, como aquela promovida pelo treinamento físico, não existem alterações nas células colágenas do coração, e com isso também não existem prejuízos funcionais, como observado para a hipertrofia patológica. ${ }^{26} \mathrm{Ten}$ do em vista essas considerações e a impossibilidade de realizar a análise da expressão gênica de marcadores de hipertrofia cardíaca, também realizamos a análise da porcentagem de colágeno presentes no miocárdio. Nesse sentido, encontramos as mesmas diferenças observadas para o diâmetro dos cardiomiócitos, onde o grupo LS apresentou maior porcentagem de fibras colágenas comparado ao CS e ao CT. Com isso, sugerimos que o L-NAME promoveu hipertrofia cardíaca e que essa hipertrofia é patológica, considerando as alterações de fibras colágenas. Além disso, é importante ressaltar, que o treinamento físico, apesar de não reverter esse quadro (LS vs. LT), também não promoveu prejuízos a morfologia cardíaca, tendo em vista que não apresentou diferenças entre os demais grupos controles (LT vs. CS e LT vs. CT), tanto para o diâmetro dos cardiomiócitos quanto para a porcentagem de colágeno.

No que diz respeito à $\mathrm{FC}$ de animais tratados com L-NAME, existem divergências na literatura. Alguns estudos apresentam resultados semelhantes ao nosso, onde o tratamento com L-NAME, apesar de aumentar a PA, não altera a $\mathrm{FC}^{7,17,27}$, outros estudos demonstram aumento ${ }^{3,4}$ e outros ainda demonstraram uma diminuição da FC de animais tratados com LNAME. ${ }^{28,29} \mathrm{O}$ estudo realizado por Dos Santos et al. ${ }^{7}$, observou que após 2 e 14 dias do inicio da hipertensão, há uma diminuição da sensibilidade do controle barorreflexo da atividade renal do nervo simpático de animais tratados com L-NAME, e dessa forma influenciar nas respostas da FC. Além disso, fatores metodológicos como, via de administração e dose do L-NAME, coleta da FC (com animais acordados ou 
anestesiados), também podem explicar as diferenças nos resultados de FC encontradas entre os estudos. No entanto, nossos resultados também demonstraram que houve redução na $\mathrm{FC}$ de animais normotensos e hipertensos treinados comparado com seus controles sedentários. Paralelamente à isso, o treinamento físico também foi eficiente em promover reduções na PA de animais hipertensos treinados comparado aos sedentários, no entanto, para os animais normotensos não houve redução na PA. Nessa perspectiva, a literatura demonstra que os mecanismos envolvidos na redução da pressão arterial promovida pelo treinamento físico, estão relacionados com fatores hemodinâmicos, neurais e humorais. ${ }^{30}$ Nesse sentido, estudos demonstram que seres humanos e animais hipertensos que são treinados, apresentam redução na PA de repouso, sendo essa redução associada à redução da atividade simpática periférica e/ou do débito cardíaco. ${ }^{31,32} \mathrm{Com}$ isso, sugere-se que a normalização do tônus simpático que está aumentado na hipertensão, pode estar associada com a bradicardia de repouso observada em animais treinados, e consequentemente com uma redução no débito cardíaco, que já foi demonstrada em animais espontaneamente hipertensos treinados. ${ }^{31,33}$ No entanto, além do controle simpático, os mecanismos de controle humoral também atuam, por meio da liberação de substâncias vasodilatadoras no controle da PA. Dentre essas substâncias vasodilatadoras, pode-se destacar a bradicinina, como um potente vasodilatador, que em condições basais parece não influenciar a PA. ${ }^{34}$ Essa relação pode explicar em partes, a redução da PA com o treinamento físico apenas para o grupo de animais hipertensos.

Além das alterações na FC, observamos redução significativa na PAS, PAD e PAM de animais hipertensos treinados comparados aos sedentários. Essa resposta de redução da PA em animais com hipertensão induzida por L-NAME está de acordo com estudos anteriores. ${ }^{21-24}$ Em nosso estudo, apesar de ter sido observada redução na pressão arterial para o grupo hipertenso treinado, esses valores não alcançaram valores equivalentes ao grupo controle, como encontrado por Husain. ${ }^{22}$ Existem evidências de que a redução pelo treinamento em animais com hipertensão induzida por L-NAME está relacionada ao aumento da atividade da síntese de NO endotelial e ao aumento das enzimas antioxidantes. ${ }^{21-24}$ Além dessas adaptações promovidas pelo treinamento, alterações na estrutura dos vasos também estão relacionadas a essa redução. ${ }^{23,24}$
Com base nos resultados encontrados, é possível que o treinamento físico tenha promovido uma melhora na função endotelial de animais com hipertensão induzida por L-NAME, os quais apresentaram menores valores de PAM comparado aos animais hipertensos sedentários. Porém no presente estudo não avaliamos a reatividade vascular, sendo essa uma sugestão para estudos futuros. Nossos resultados também demonstraram uma atenuação da pressão arterial em animais hipertensos treinados, o que sugere que o treinamento físico por um período menor (quatro semanas) é suficiente em promover adaptações ao sistema cardiovascular a fim de atenuar a hipertensão. No entanto, tornam-se necessários estudos que analisem a reatividade vascular em animais tratados com L-NAME e treinados.

Além de promover o aumento da PA, a administração de L-NAME associada ao exercício pode promover alterações morfológicas no sistema cardiovascular $^{10}$ e nos rins ${ }^{.25}$ Dessa forma, é provável que outros fatores, além do endotélio, possam participar das alterações observadas na PA. Nesse sentido, também investigamos essas possíveis alterações, promovidas tanto pela administração de L-NAME quanto pelo treinamento físico. Em relação à hipertrofia cardíaca, observamos que o treinamento físico não promoveu alterações para os animais tratados com solução salina. No entanto, para o grupo LT, houve um maior peso absoluto e relativo do coração, comparado ao CT. Esses achados sugerem que uma possível hipertrofia cardíaca ocorreu devido à administração de L-NAME, e não devido ao treinamento. Para que ocorra hipertrofia cardíaca fisiológica, ou seja, aquela promovida pelo treinamento físico, é necessário que haja uma sobrecarga adequada para observar esses efeitos. O protocolo de treinamento utilizado neste estudo foi conduzido sem sobrecarga adicional, além do peso do animal e do tempo na primeira semana de adaptação. Além disso, observamos que houve uma redução de marcadores inflamatórios (áreas contendo neutrófilos e macrófagos) para o grupo LT comparado ao LS, o que demonstra uma contribuição importante do treinamento na regulação dessas variáveis, que podem contribuir para a redução da PA.

\section{Conclusão}

Os achados do presente estudo demonstraram que este protocolo de indução de hipertensão foi eficiente e que o treinamento físico, além de promover a 
bradicardia de repouso em animais normotensos e hipertensos, também foi eficiente em reduzir a PAM de animais hipertensos em relação à animais hipertensos sedentários. Além disso, a morfologia cardíaca confirmou a hipertrofia cardíaca promovida pelo LNAME e com aumento da porcentagem de colágeno indicando a hipertrofia patológica. A hipertensão promovida por L-NAME promoveu o aumento das áreas de colágeno e o treinamento físico contribuiu para uma redução dessas áreas. Dessa forma, foi confirmada a hipótese de que este protocolo de treinamento físico não foi capaz de promover a hipertrofia fisiológica e que este protocolo de indução de hipertensão induz a hipertrofia cardíaca.

\section{Agradecimentos}

À Fundação Araucária e CNPq pelo apoio financeiro.

Conflito de interesses: os autores declaram não haver conflito de interesses.

\section{Referências}

1. Bartunek J, Weinberg EO, Tajima M, Rohrbach S, Katz SE, Douglas PS, et al. Chronic N(G)-nitro-L-arginine methyl esterinduced hypertension : novel molecular adaptation to systolic load in absence of hypertrophy. Circulation. 2000;101:423-9.

2. Hu CT, Chang HR, Hsu YH, Liu CJ, Chen HI. Ventricular hypertrophy and arterial hemodynamics following deprivation of nitric oxide in rats. Life Sci. 2005;78:164-73.

3. Rossi BR, Mazer D, Silveira LC, Jacinto CP, Di Sacco TH, Blanco $\mathrm{JH}$, et al. Physical exercise attenuates the cardiac autonomic deficit induced by nitric oxide synthesis blockade. Arq Bras Cardiol. 2009;92:31-8.

4. Souza HC, De Araujo JE, Martins-Pinge MC, Cozza IC, Martins-Dias DP. Nitric oxide synthesis blockade reduced the baroreflex sensitivity in trained rats. Auton Neurosci. 2009;150:38-44.

5. Ribeiro MO, Antunes E, de Nucci G, Lovisolo SM, Zatz R. Chronic inhibition of nitric oxide synthesis. A new model of arterial hypertension. Hypertension. 1992 Sep;20(3):298-303.

6. Moncada S, Higgs A. The L-arginine-nitric oxide pathway. N Engl J Med. 1993; 329:2002-12.

7. Dos Santos FM, Martins Dias DP, da Silva CA, Fazan R, Jr., Salgado HC. Sympathetic activity is not increased in L-NAME hypertensive rats. Am J Physiol, Regul Integr Comp Physiol. 2010;298:89-95.

8. Akuzawa N, Nakamura T, Kurashina T, Saito Y, Hoshino J, Sakamoto $\mathrm{H}$, et al. Antihypertensive agents prevent nephrosclerosis and left ventricular hypertrophy induced in rats by prolonged inhibition of nitric oxide synthesis. Am J Hypertens. 1998;11:697-707.

9. Vrankova S, Parohova J, Barta A, Janega P, Simko F, Pechanova O. Effect of nuclear factor kappa B inhibition on LNAME-induced hypertension and cardiovascular remodelling. J Hypertens. 2010;28: (Suppl 1) S45-9.
10. Souza HC, Penteado DM, Martin-Pinge MC, Barbosa Neto O, Teixeira Vde P, Blanco JH, et al. Nitric oxide synthesis blockade increases hypertrophy and cardiac fibrosis in rats submitted to aerobic training. Arq Bras Cardiol. 2007;89:88-93, 9-104.

11. Cardoso AM, Martins CC, Fiorin Fda S, Schmatz R, Abdalla $\mathrm{FH}$, Gutierres J, et al. Physical training prevents oxidative stress in L-NAME-induced hypertension rats. Cell Biochem Funct. 2013;31:136-51.

12. Martins-Pinge MC, Becker LK, Garcia MR, Zoccal DB, Neto $\mathrm{RV}$, Basso LS, et al. Attenuated pressor responses to amino acids in the rostral ventrolateral medulla after swimming training in conscious rats. Auton Neurosci. 2005;122:21-8.

13. Bertagnolli M, Schenkel PC, Campos C, Mostarda CT, Casarini $\mathrm{DE}$, Bello-Klein A, et al. Exercise training reduces sympathetic modulation on cardiovascular system and cardiac oxidative stress in spontaneously hypertensive rats. Am J Hypertens. 2008;21:1188-93.

14. Bermudes AM, Vassallo DV, Vasquez EC, Lima EG. Ambulatory blood pressure monitoring in normotensive individuals undergoing two single exercise sessions: resistive exercise training and aerobic exercise training. Arq Bras Cardiol. 2004;82:65-71, 57-64.

15. Forjaz CL, Tinucci T, Ortega KC, Santaella DF, Mion D, Jr., Negrao CE. Factors affecting post-exercise hypotension in normotensive and hypertensive humans. Blood Press Monit. 2000;5:255-62.

16. Bell D, Campbell M, Wang X, Earle JA, Cosby SL, McDermott BJ. Adrenomedullin gene delivery is cardio-protective in a model of chronic nitric oxide deficiency combining pressure overload, oxidative stress and cardiomyocyte hypertrophy. Cell Physiol Biochem. 2010;26:383-94.

17. Biancardi VC, Bergamaschi CT, Lopes OU, Campos RR. Sympathetic activation in rats with L-NAME-induced hypertension. Braz J Med Biol Res. 2007;40:401-8.

18. Paulis L, Pechanova O, Zicha J, Barta A, Gardlik R, Celec P, et al. Melatonin interactions with blood pressure and vascular function during L-NAME-induced hypertension. J Pineal Res. 2010;48:102-8.

19. Simko F, Pechanova O, Pelouch V, Krajcirovicova K, Celec P, Palffy R, et al. Continuous light and L-NAME-induced left ventricular remodelling: different protection with melatonin and captopril. J Hypertens. 2010;28: (Suppl 1) S13-8.

20. Zicha J, Kunes J, Vrankova S, Jendekova L, Dobesova Z, Pinterova M, et al. Influence of pertussis toxin pretreatment on the development of L-NAME-induced hypertension. Physiol Res. 2009;58:751-5.

21. Husain K. Interaction of exercise training and chronic NOS inhibition on blood pressure, heart rate, NO and antioxidants in plasma of rats. Pathophysiology. 2003;10:47-56.

22. Husain K. Exercise conditioning attenuates the hypertensive effects of nitric oxide synthase inhibitor in rat. Mol Cell Biochem. 2002;231:129-37.

23. Kuru O, Senturk UK, Demir N, Yesilkaya A, Erguler G, Erkilic $M$. Effect of exercise on blood pressure in rats with chronic NOS inhibition. Eur J Appl Physiol. 2002;87:134-40.

24. Kuru O, Senturk UK, Kocer G, Ozdem S, Baskurt OK, Cetin A, et al. Effect of exercise training on resistance arteries in rats with chronic NOS inhibition. J Appl Physiol. 2009;107:896902.

25. Raij L. Nitric oxide in hypertension: relationship with renal injury and left ventricular hypertrophy. Hypertension. 1998;31:189-93. 
26. Mill J, Vassallo DV. Hipertrofia cardíaca. Rev Bras Hipertens. 2001;8:63-75.

27. Miao C, Shen F, Su D. Blood pressure variability is increased in genetic hypertension and L-NAME induced hypertension. Acta Phamacol Sin. 2001;22:137-40.

28. Reinhart GA, Lohmeier TE, Mizelle HL. Temporal influence of the renal nerves on renal excretory function during chronic inhibition of nitric oxide synthesis. Hypertension. 1997;29:199204.

29. Scrogin KE, Hatton DC, Chi Y, Luft FC. Chronic nitric oxide inhibition with L-NAME: effects on autonomic control of the cardiovascular system. Am J Physiol. 1998;274:367-74.

30. Irigoyen MC, De Angelis K, Schaan BDA, Fiorino P, Michelini LC. Exercício físico no diabetes melito associado a hipertensão arterial sistêmica. Rev Bras Hipertens. 2003;10:109-16.
31. Veras-Silva AS, Mattos KC, Gava NS, Brum PC, Negrao CE, Krieger EM. Low-intensity exercise training decreases cardiac output and hypertension in spontaneously hypertensive rats. Am J Physiol. 1997;273:2627-31.

32. Hagberg JM, Montain SJ, Martin WH, 3rd, Ehsani AA. Effect of exercise training in 60- to 69-year-old persons with essential hypertension. Am J Cardiol. 1989;64:348-53.

33. Gava NS, Veras-Silva AS, Negrao CE, Krieger EM. Low-intensity exercise training attenuates cardiac beta-adrenergic tone during exercise in spontaneously hypertensive rats. Hypertension. 1995;26:1129-33.

34. Brandão AF, Martins-Pinge MC. Alteração do óxido nítrico na função cardiovascular pelo treinamento físico. Semina. 2007;28:53-68. 